\title{
Action du sulfite de sodium sur la concentration en composés organohalogénés et sur l'activité mutagène de solutions chlorées de substances humiques Effects of sodium sulfite on the concentration of organohalogenated compounds and on the mutagenic activity of chlorinated solutions of humic substances
}

\author{
C. Morlay, J. De Laat, M. Dore, Y. Courtois, A. Montiel, B. Welte et N. Houel
}

Volume 5, numéro 3, 1992

URI : https://id.erudit.org/iderudit/705135ar

DOI : https://doi.org/10.7202/705135ar

\section{Aller au sommaire du numéro}

\section{Éditeur(s)}

Université du Québec - INRS-Eau, Terre et Environnement (INRS-ETE)

\section{ISSN}

0992-7158 (imprimé)

1718-8598 (numérique)

\section{Découvrir la revue}

Citer cet article

Morlay, C., De Laat, J., Dore, M., Courtois, Y., Montiel, A., Welte, B. \& Houel, N. (1992). Action du sulfite de sodium sur la concentration en composés organohalogénés et sur l'activité mutagène de solutions chlorées de substances humiques. Revue des sciences de l'eau / Journal of Water Science, 5(3), 335-352. https://doi.org/10.7202/705135ar

\section{Résumé de l'article}

Cette étude a eu pour but de déterminer l'effet d'un traitement par le sulfite de sodium sur la concentration en composés organohalogénés totaux (TOX) et sur l'activité mutagène de solutions chlorées de substances humiques d'origine aquatique (SHA), après avoir cherché à préciser l'influence du pH et du temps sur la concentration en TOX.

Les résultats obtenus à partir d'échantillons chlorés de SHA en absence de chlore résiduel ont permis de mettre en évidence une diminution de la concentration en composés organohalogénés totaux, soit par stockage en milieu neutre ou basique, soit par addition de sulfite de sodium. L'intensité de cette réduction de la concentration en TOX augmente avec le $\mathrm{pH}$, le temps de réaction et la dose de sulfite de sodium introduite.

Les résultats obtenus à partir d'échantillons contenant du chlore libre indiquent que seule une déchloration totale avec un excès de sulfite de sodium peut conduire, en milieu neutre, à une diminution de l'activité mutagène et de la concentration en TOX des solutions diluées de SHA. La comparaison des pourcentages d'abattement obtenus sur le paramètre TOX et sur l'activité mutagène indique que la diminution de la génotoxicité par déchloration totale est due à l'action du sulfite sur des composés mutagènes non chlorés ou sur des composés chlorés fortement mutagènes et ne représentant qu'une très faible fraction du TOX. 


\section{Action du sulfite de sodium sur la concentration en composés organohalogénés et sur l'activité mutagène de solutions chlorées de substances humiques**}

\section{Effects of sodium sulfite on the concentration of organo- halogenated compounds and on the mutagenic activity of chlorinated solutions of humic substances}

C. MORLAY1, J. DE LAAT', M. DORE"1, Y. COURTOIS?,

A. MONTIEL 3 , B. WELTE 3, N. HOUEL 4

Recu le 15 jarvier 1991, accepté pour pubication le 10 decembre 1991*.

SUMMARY

It is a well known fact that numerous organohalogenated compounds are formed during the chlorination (preexidation or final disinfection) of drinking water. Some of these compounds have been shown to be mutagenic. Recent studies have suggested that a treatment with oxygenated derivatives of SIV $\left(\mathrm{SO}_{2}, \mathrm{NaHSO}_{3}\right.$ and $\left.\mathrm{Na}_{2} \mathrm{SO}_{3}\right)$ could reduce the genotoxicity of chlorinated drinking water.

The general aim of this study was to determine the effect of dechlorination treatments on the mutagenic activity of chlorinated drinking water. The following experiments were carried out in order to point out the effect of a treatment with sodium sulfite on the concentration of total organohalogenated compounds (TOX) and on the mutagenic activity of chlorinated dilute solutions of Aquatic Hamic Sabstances (AHS).

At first, the effects of pH, sodium sulfite dose and contact time on TOX concentration were iavestigated. Then, the importance of the dechlorination rate (partial or complete) on TOX concentration and also on the mutagenic activity could be studied.

Experimental

Aquatic Humic Substances (natural mixture of fulvic and humic acids) were dissolved in phosphate-buffered ultra-pure water at 5 and $15 \mathrm{mg} \mathrm{I-1}$ concentrations (pH 6.1 and 6.9 respectively). Stock solutions of chlorine were

1. Laboratoire de Chimie de l'Eau et des Muisances - UA CNRS no 1468.

Ecole Supérieure d'Ingénieurs de Poitiers, 40, avenue du Recteur Pineau, 86022 Poitiers cedex, Tél: 49.45.39.15.

2. Laboratoire d'Hygiène de la Ville de Paris, 11 , rue Georges Eastman, 75013 Paris, Th́ : (1) 45.82 .80 .50 .

3. Société Anonyme de Gestion des Eaux de Paris, 9 rue Schoelcher, 75014 Paris, Tél : (1) 40.48.99.20.

4. Agence de Bassin Seine-Normandie, 51, rue Salvador Allende, 92027 Nanterre cedex, Tél : (1) 47.76.44.24.

- Les commentaires seront recus jusqu'au 30 mars 1993.

- Communication présentée à la joumée "Jeunes chercheurs" du Gruttee, le 28 septembre 1990 à Poitiers. 
prepared in the laboratory and titrated by iodometry. Chlorination and dechlorination treatments were carried out in headspace-free bottles, at $20 \pm$ $1^{\circ} \mathrm{C}$ in the dark. Residual chiorine was determined by spectrophotometric measurements at $510 \mathrm{~nm}$, following the colorimetric method using $N, N$-diethylphenylene-1,4-diamine (DPD). To avoid the slow oxidation of $S_{j y}$ into $S_{y_{1}}$ by dissolved oxygen, the sodium sulfite solutions were prepared freshly before use. TOX concentrations were measured using a DOHRMAN DX-20 TOX analyser equipped with a MC-1 microcoulometric cell and with an AD-2 adsorption module. Before analysis, the residual chlorine was neutralized with sodium thiosulfate and samples were aciditied to $\mathrm{pH} 1.4$.

The mutagenic activity was determined using acetone-dichloromethane extracts (AMBERLITE XAD-8 and XAD-2 resins) of the aqueous samples of chlorinated and dechlorinated solutions of AHS, acidified to $\mathrm{pH} 2.0$ before extraction. The mutagenicity tests were carried ouf on TA 98 and TA 100 tester strains, following the method described by MARON and AMES (1983).

Results

- Effect of $\mathrm{pH}$, addition of sodium sulfite and storage time on the roX concentration

The experiments carried out with dilute solutions of AHS ([AHS] $=5 \mathrm{mg} \mathrm{\textrm {t } ^ { - 1 }}$; $\mathrm{DOC}=2.5 \mathrm{mg} \mathrm{C}^{-1} ; \mathrm{pH}=6.1$ ) showed a linear relationship between $\mathrm{TOX}$ production and chlorine consumption in the range $0-2.0 \mathrm{mg} \mathrm{Cl}_{2} \mathrm{I}^{-1}$ (fig.2). $15 \%$ of the chlorine demand was incorporated as organic chlorine in molecules.

Experiments performed on solutions containing no residual free chlorine showed that organohalogenated compounds could be partially destroyed upon storage at neutral or basic pH (table 1). Reductions in TOX concentrations of $10 \%$ at pH 6.1-8.5 in 24 hours and of $20 \%$ at pH 11.5 in 2 hours were observed. This was enhanced by increasing the storage time.

The addition of sodium sulfite $\left(100 \mu \mathrm{mol} \mathrm{I}^{-1}\right)$ in solutions containing no residual free chlorine significantly reduced the TOX concentration (10\% in 2 hours at pH 6.1-8.5; table 1). This reduction was enhanced by increasing sulfite dose and storage time and by increasing $\mathrm{pH}(30 \%$ in 2 hours at pH 11.5). Furthermore, at a given pH value and for a reaction time of 2 hours, the decrease in TOX concentration was larger in presence of sulfite.

\section{- Effect of a dechlorination treatment on the TOX concentration}

As shown in figure 3, a dechlorination treatment (reduction of the residual free chlorine concentration) with sodium sulfite could significantly reduce the TOX concentration of the dilute solutions of AHS at pH 6.1 only if an excess of the dechlorinating agent was added. This effect was enhanced by increasing the excess of sulfite but nevertheless seemed to be limited (less than $15 \%$ of reduction for the highest doses used; table 2).

The free chlorine residuals measured after a 2 hours partial dechlorination confirmed the stoichiometric factor of $1 \mathrm{~mole} / \mathrm{mole}$ for the reaction between chlorine and sodium sulfite.

- Effect of a dechlorination treatment on the mutagenic activity and on the TOX concentration

The dechlorination treatments were carried out on chlorinated dilute solutions

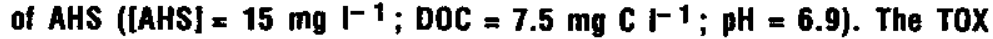
concentrations were measured on aqueous solutions and mutagenicity tests were performed on the corresponding acetone-dichloromethane extracts folfowing a solvent exchange (dimethylsulfoxide). The results obtained showed again that only a total dechlorination treatment could reduce the TOX 
concentration of the aqueous chlorinated solutions and was able to destroy a significant part of the mutagenic activity of the extracts (table 3 and fig. 4).

Although the effect of sulfite on TOX concentration seemed limited (less than $7 \%$ reduction for the highest sulfite dose tested), the reduction in the genotoxicity was more important when the excess of sulfite was increased. No correlation between the TOX concentration and the mutagenic activity could be established. The mutagenic compounds destroyed by sodium sulfite do not appear to be organohalogenated ones. If they are, they are present at trace levels and thus are extremely potent and account for a very little part of the TOX concentration.

Key-words : chlorination, dechlorination, sodium sulfite, aquatic humic substances, total organohalogenated compounds, mutagenic activity.

\section{RÉSUMÉ}

Cette étude a eu pour but de déterminer l'effet d'un traitement par le sulfite de sodium sur la concentration en composés organohalogénés totaux (TOX) et sur l'activité mutagène de solutions chlorées de substances humiques d'origine aquatique (SHA), après avoir cherché à préciser l'influence du pH et du temps sur la concentration en ToX.

Les résultats obtenus a partir d'échantillons chlorés de SHA en absence de chlore résiduel ont permis de mettre en évidence une diminution de la concentration en composés organohalogénés totaux, soit par stockage en milieu neutre ou basique, soit par addition de sulfite de sodium. L'intensité de cette réduction de la concentration en TOX augmente avec le pH, le temps de réaction et la dose de sulfite de sodium introduite.

Les résultats obtenus a partir d'échantillons contenant du chlore libre indiquent que seule une déchloration totale avec un excès de sulfite de sodium peut conduire, en milieu neutre, à une diminution de l'activité mutagène et de la concentration en TOX des solutions diluées de SHA. La comparaison des pourcentages d'abattement obtenus sur le paramètre TOX et sur l'activité mutagène indique que la diminution de la génotoxicité par déchloration totale est due à l'action du sulfite sur des composés mutagènes non chlorés ou sur des composés chlorés fortement mutagènes et ne représentant qu'une très faible traction du TOX.

Mots clés : chloration, déchloration, sulfite de sodium, substances humiques aquatiques, composés organohalogéness totaux, activité mutagène.

\section{INTRODUCTION}

II est maintenant bien établi que la chloration des eaux à potabiliser (préoxydation ou désinfection finale) conduit à la formation de nombreux composés organohalogénés (GLAZE et al., 1980 ; GLAZE et al., 1979 ; ROOK, 1974 ; BELLAR et al., 1974) parmi lesquels un certain nombre présente des propriétés mutagènes (HORTH, 1989 ; KRONBERG et al., 1987 ; FIELDING et HORTH, 1986). Par ailleurs, des études récentes semblent montrer qu'une diminution de l'activité mutagène des eaux chlorées peut être obtenue par un 
traitement mettant en œuvre des dérivés oxygénés du soufre tétravalent $\left(\mathrm{SO}_{2}\right.$, $\mathrm{NaHSO}_{3}$ et $\mathrm{Na}_{2} \mathrm{SO}_{3}$ ) (FAWELL et al., 1986 ; WILCOX et DENNY, 1985 ; CHEH ef al., 1980). Ces composés sont introduits avant distribution dans certaines eaux potables de la Région Parisienne afin de lutter contre les problèmes de goûts et odeurs dus à la présence de chlore libre résiduel à une teneur trop importante mais cependant nécessaire compte tenu des temps de séjour de l'eau dans le réseau (MAQUENNEHAN et ClAUSE, 1987 ; ANSELME et al., 1987 ; JESTIN et al., 1986). La diminution de l'activité génotoxique observée peut être en partie expliquée par une dégradation des composés organohalogénés mutagènes résultant d'un processus d'hydrolyse (CROUE et RECKHOW, 1989 ; KOPFLER et al., 1985 ; HOLMBOM et al., 1984 ; KRINGSTAD et al., 1983) ou de l'action de composés réducteurs et en particulier des dérivés oxygénés du soufre tétravalent (SIV) (CROUE et RECKHOW, 1989 ; FAM, 1986 ; TREHY et BIEBER, 1981).

Ces travaux, qui s'inscrivent dans le cadre plus général d'une étude relative à l'influence des traitements de déchloration sur l'activité mutagène des eaux de distribution chlorées, ont eu pour but de préciser l'effet d'un traitement par le sulfite de sodium sur la concentration en composés organohalogénés totaux (paramètre TOX) et sur l'activité mutagène de solutions chlorées de substances humiques d'origine aquatique. Ces composés, naturellement présents dans les eaux de surface, sont reconnus comme étant les principaux précurseurs des composés organohalogénés (CROUE, 1987 ; MEIER et al., 1983 ; OLIVER, 1978) et mutagènes (HORTH, 1989 ; FIELDING et HORTH, 1986 ; MEIER et al., 1983) formés lors de la chloration.

Au cours de ce travail, réalisé au laboratoire, on aura dans un premier temps étudié l'influence du pH, de la dose de sulfite de sodium et du temps de contact sur le paramètre TOX afin de préciser les conditions optimales de conservation des échantillons et de traitement par les dérivés du SIV. Dans une deuxième partie, on s'attachera à montrer l'influence du taux de déchloration (déchloration partielle ou totale) sur la concentration en TOX et sur l'activité mutagène.

\section{2 - MATÉRIEL ET MÉTHOdES}

Toutes les solutions mises en œuvre au cours de cette étude ont été préparées à partir d'eau ultra-pure fournie par un ensemble Millipore (Milli $Q+$ Milli RO-4; résistivité $=18 \mathrm{M} \Omega \mathrm{cm}$; Carbone Organique Dissous $=50-100 \mu \mathrm{g}$ $(-1)$. Les produits chimiques employés sont de qualité analytique. Les solvants ont été distillés au laboratoire (méthanol, éther) ou sont de qualité PESTIPUR (acétone et dichlorométhane pour l'extraction des pesticides et leur dosage par CPG avec détecteur à capture d'électrons ; pureté minimum de $99,7 \%$ et $99,95 \%$ respectivement). Les réactions ont été conduites à $20 \pm$ $1^{\circ} \mathrm{C}$ et les stockages effectués à l'obscurité et à la même température. 


\subsection{Solutions de substances humiques aquatiques}

Les substances humiques utilisées ont été obtenues à partir d'une eau de barrage, selon le protocole d'extraction sur résine macroporeuse XAD-8 et de conditionnement décrit par THURMAN et MALCOLM (1981). Les étapes finales de séparation (précipitation de la fraction humique en milieu acide) n'ont pas été réalisées, afin de travailler sur le mélange d'acides humique et fulvique représentatif de la composition de l'eau en substances humiques (acide humique : $10 \%$, acide fulvique : $90 \%$ ). L'analyse élémentaire, réalisée par le Service Central d'Analyse du CNRS, a révélé les pourcentages massiques suivants: $\mathrm{C}=49,4 ; \mathrm{H}=5,2 ; \mathrm{O}=39,4 ; \mathrm{N}=2,1 ; \mathrm{S}=1,3 ;$ résidu analysé $=1,6$.

L'influence de différents traitements sur la concentration en TOX a été étudiée à partir de solutions de SHA à $5 \mathrm{mg} \mathrm{l-1}$. Pour suivre, lors d'une déchloration, l'évolution de la concentration en TOX parallèle à celle de l'activité mutagène, des solutions de SHA à $15 \mathrm{mg} \mathrm{I}^{-1}$ ont été utilisées. Pour disposer, au cours de chacune des deux études, de solutions constituées du même lot initial de SHA et présentant une similitude maximale au niveau de la concentration en matière organique, des solutions mères de SHA à $100 \mathrm{mg} \mathrm{t}^{-1}$ (étude du TOX seul) et $1,5 \mathrm{~g} \mathrm{t}^{-1}$ (étude de l'activité mutagène et du TOX) ont été préparées. Dans le second cas, il a été nécessaire d'utiliser un peu d'une solution de soude à $0,5 \mathrm{~mol} \mathrm{I}^{-1}$ pour solubiliser les $\mathrm{SHA}$ (à $\left.\mathrm{pH} \approx 7,0\right)$. Les solutions filles à 5 et $15 \mathrm{mg}^{-1}$ ont été obtenues par dilution des solutions mères correspondantes et tamponnées à l'aide de sels de phosphates $(\mathrm{pH}=$ 6,$1 ; \mu=0,04 \mathrm{~mol} \mathrm{I}^{-1}$ et $\mathrm{pH}=6,9 ; \mu=0,01 \mathrm{~mol} \mathrm{I}^{-1}$ respectivement).

\subsection{Solutions d'hypochlorite de sodium}

Une solution mère d'hypochlorite de sodium ( $\mathrm{NaClO}$ ) a été préparée au laboratoire par réaction entre de l'acide chlorhydrique concentré et du permanganate de potassium, le chlore gazeux libéré étant piégé dans une solution de soude à environ $1 \mathrm{~mol}^{-1}$. Avant chloration, cette solution mère $\left(\approx 20 \mathrm{~g} \mathrm{Cl}_{2} \mathrm{I}^{-1}\right)$ a été diluée et son $\mathrm{pH}$ ajusté à $8,5 \pm 0,1$ par ajout d'acide chlorhydrique concentré. Le titre de la solution obtenue a été déterminé par dosage iodométrique.

\subsection{Solutions de sulfite de sodium}

Les solutions de sulfite de sodium (sulfite de sodium anhydre, produit Fluka, Bio-Chemika Micro-Select, pureté supérieure à 99,5\%) ont été préparées juste avant leur utilisation de façon a éviter toute modification de leur titre par oxydation lente en présence d'oxygène dissous (WATTS et ADAMS, 1983 ; FULLER et CRIST, 1941).

\subsection{Méthodes analytiques}

\subsubsection{Mesure du chlore résiduel}

Le chlore résiduel a été dosé par spectrophotométrie à $510 \mathrm{~nm}$ (spectrophotomètre UV/visible Varian DMS 90) selon la méthode colorimétrique à la 
N,N-diéthylphénylène-1,4-diamine (DPD) (norme AFNOR « eaux méthodes d'essai » T 90-038 d'avril 1984, pour des concentrations en chlore total de 0,03 à $\left.5 \mathrm{mg} \mathrm{Cl}_{2} \mathrm{I}^{-1}\right)$.

\subsubsection{Détermination des concentrations en composés organohalogénés totaux}

Les concentrations en composés organohalogénés totaux (TOX) ont été déterminées à l'aide d'un analyseur Dohrmann DX-20 équipé d'un module d'adsorption AD-2 et d'un microcoulomètre MC-1.

Après neutralisation du chlore résiduel (pas d'excès de réducteur) par une solution de thiosulfate de sodium à 0,1 mol $^{-1}$, l'étape d'adsorption sur charbon actif en poudre ( 2 microcolonnes en série contenant chacune $40 \pm 2 \mathrm{mg}$ de CAP) permet de concentrer les composés organohalogénés contenus dans les $100 \mathrm{ml}$ de l'échantillon à analyser. Celui-ci est auparavant acidifié à pH 1,4 $\pm 0,1\left(\mathrm{HNO}_{3}\right.$ concentré) afin d'améliorer l'adsorbabilité des composés organiques. Lors des deux études, l'acidification des échantillons à pH 1,4 a toujours été réalisée immédiatement après neutralisation du chlore résiduel par le thiosulfate ou, dans le cas d'une chloration sans chlore résiduel ou d'une déchloration totale, au terme du temps de réaction choisi. Après lavage de chaque colonne par une solution de nitrate de potassium pour éliminer les halogénures inorganiques, le CAP est pyrolysé à $800^{\circ} \mathrm{C}$ et les halogénures d'hydrogène formés sont dosés par microcoulométrie. Les performances du microcoulomètre sont régulièrement vérifiées par injection, directement dans la cellule de mesure, d'une solution étalon de chlorure sous forme de chlorure de sodium $(\mathrm{NaCl})$.

La concentration en TOX d'un échantillon, exprimée en masse équivalente de chlore atomique par litre, est calculée en tenant compte de la quantité d'halogénures contenue dans chacune des deux microcolonnes de CAP, de la quantité moyenne d'halogénures contenue dans une colonne de CAP lavée seulement par la solution de $\mathrm{KNO}_{3}$ et du volume de l'échantillon analysé. Pour un échantillon de $100 \mathrm{ml}$, la gamme utile de mesure s'étend de 5 à $500 \mu \mathrm{g} \mathrm{Cl}$ $\mathrm{I}^{-1}$ et la gamme optimale de 10 à $250 \mu \mathrm{g} \mathrm{Cl}{ }^{-1}$. Dans ce dernier cas, la précision de la mesure est de $2 \%$. Tous les échantillons d'une série ont été analysés le même jour.

\subsubsection{Détermination de l'activité mutagène}

L'activité mutagène des solutions de SHA à $15 \mathrm{mg} \mathrm{l}^{-1}$, chlorées puis déchlorées par le sulfite de sodium à différents taux, a été déterminée sur les extraits correspondants. Ces extraits ont été obtenus à l'aide de résines organiques macroporeuses Amberlite XAD-8 et XAD-2 (Rohm and Haas). Avant utilisation, elles ont été conditionnées par plusieurs lavages à l'eau ultra-pure pour éliminer les fines particules, par des extractions en soxhlet avec du méthanol (60 heures), de l'éther (72 heures), du dichlorométhane (48 heures), puis par rinçage avec de l'eau ultra-pure.

Les échantillons, préalablement acidifiés à pH 2,0 $\pm 0,1(\mathrm{HCl}$ concentré) percolent en flux ascendant à raison de 2,0 $\pm 0,2 \mathrm{I} \mathrm{h}^{-1}$ (soit, pour $100 \mathrm{ml}$ de chacune des résines, $10 \pm 1 \mathrm{~V} / \mathrm{N} / \mathrm{H}$ ). La résine XAD-8 est placée en amont. Le 
lit de résine a un diamètre de $4,0 \mathrm{~cm}$ et une hauteur de $16,0 \mathrm{~cm}$. La phase d'adsorption dure environ 22 heures. Lorsque l'étape d'adsorption est terminée, l'eau interstitielle est en grande partie éliminée par aspiration à l'aide d'une trompe à eau. L'élution est alors pratiquée à contre-courant de la filtration avec 1 volume d'acétone (temps de contact de 10 heures environ puis passage à $0,5 \mathrm{~V} / \mathrm{N} / \mathrm{H}$, soit $100 \mathrm{ml} \mathrm{h}^{-1}$ ) puis 2 volumes de dichlorométhane (même débit que précédemment - pas d'interruption de l'élution). L'élution dure environ 6 heures. Seule la phase la plus dense de l'éluat (dichlorométhane essentiellement) est récupérée puis séchée sur du sulfate de sodium anhydre de qualité Pestipur. La reconcentration (de 500 à $30 \mathrm{ml}$ environ) est opérée par évaporation du solvant dans un appareil Kuderna-Danish (température du bain $=65 \pm 1^{\circ} \mathrm{C}$ ) équipé d'une colonne Snyder.

Les tests de mutagénèse ont été réalisés au Laboratoire d'Hygiène de la Ville de Paris selon le protocole décrit par MARON et AMES (1983) après un échange de solvant (diméthyisulfoxyde). Les souches Salmonella typhimurium TA 98 et TA 100 ont été utilisées avec ou sans activation métabolique par la fraction S9 mix (homogénat de foie de rat Sprague Dawley traité par l'Aroclor 1254, complémenté en cofacteurs). Pour chaque échantilion, la réponse des souches a été déterminée sur 5 doses avec 2 boîtes par dose. L'échantillon est considéré mutagène si une relation dose-effet linéaire est obtenue et si, pour au moins une des doses testées, un doublement du nombre de révertants par rapport au taux de révertants spontanés est observé. La valeur de la pente calculée dans la partie linaire de la courbe dose-effet permet alors de quantifier l'activité mutagène de l'échantillon considéré. Des témoins positifs et négatifs permettent de vérifier la réponse des souches.

\section{3 - RESULTATS ET DISCUSSIONS}

\subsection{Influence de différents traitements sur la concentration en composés organohalogénés totaux}

Pour minimiser le volume d'air présent et ainsi éviter les pertes de composés organohalogénés volatils, les échantillons de $100 \mathrm{ml}$ de solution de SHA à $5 \mathrm{mg} \mathrm{I}^{-1}$ (COD $=2,5 \mathrm{mg} \mathrm{Cl}^{-1} ; \mathrm{pH}=6,1 ; \mu=0,04 \mathrm{~mol} \mathrm{I}^{-1}$ ) ont été chlorés et déchlorés dans des fioles jaugées de $100 \mathrm{ml}$ en verre et ensuite bouchées.

\subsubsection{Taux de chloration et production de TOX}

Les échantillons ont été chlorés à $\mathrm{pH}=6,1$ à des taux de 0,60 à $7,54 \mathrm{mg}$ $\mathrm{Cl}_{2} \mathrm{~F}^{-1}$ (soit de 0,12 à $1,51 \mathrm{mg} \mathrm{Cl} / \mathrm{mg} \mathrm{SHA}$ ). Le chlore résiduel a été mesuré après 15 heures et neutralisé par le thiosulfate de sodium. Les échantillons ont alors été immédiatement acidifiés $(\mathrm{pH} 1,4 \pm 0,1)$ et leur concentration en TOX mesurée le jour même. 
Les résultats obtenus (fig. 1) montrent que :

- le chlore libre est absent des échantillons traités à des taux inférieurs à $1,15 \mathrm{mg} \mathrm{Cl}{ }^{1-1}$. Pour les expériences suivantes, des taux de chloration inférieurs à cette valeur ont été mis en œuvre lorsque l'absence de chlore résiduel après 15 heures de réaction était souhaitée ;

- la production de TOX augmente lorsque le taux de chloration augmente ; la courbe de production de TOX suit celle de la consommation en chlore. La représentation des concentrations en TOX en fonction des taux de chlore consommé donne alors une droite de coefficient de corrélation supérieur à 0,99 . Le calcul de la pente de cette droite indique que dans les conditions opératoires choisies $15 \%$ du chlore consommé sont incorporés dans des composés organohalogénés.

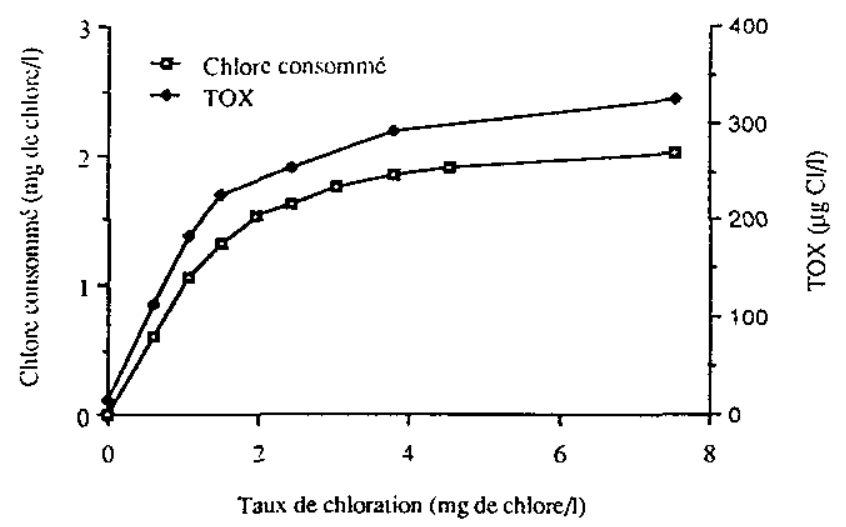

Figure 1 Influence du taux de chloration appliqué sur la consommation en chlore et la production de $\operatorname{TOX}\left([S H A]=5 \mathrm{mg} \mathrm{l}^{-1} ; \mathrm{pH}=6,1 ; 20 \pm 1^{\circ} \mathrm{C}\right.$; temps de réaction $=15$ heures).

Effect of the applied chlorine dose on the chlorine consumption and on the rOX production ([AHS] $=5 \mathrm{mg}^{-1} ; \mathrm{pH}=6.1 ; 20 \pm 1^{\circ} \mathrm{C} ; 15$ hours reaction time).

\subsubsection{Influence du pH du milieu, de l'addition de sulfite de sodium et du temps de stockage sur la concentration en TOX}

Trois séries d'échantillons de SHA ont été chlorées à pH 6,1 et à un taux de $1,13 \mathrm{mg} \mathrm{Cl} \mathrm{I}^{-1}$. Après 15 heures (chlore résiduel nul) la concentration moyenne en TOX était de $204 \pm 4 \mu \mathrm{g} \mathrm{Cl} \mathrm{Cl}^{-1}$ et le $\mathrm{pH}$ était inchangé. Le $\mathrm{pH}$ des échantillons de la première série a alors été ajusté à $2,0 \quad\left(\mathrm{H}_{3} \mathrm{PO}_{4}\right.$ concentré), 8,5 ou 11,5 ( $\mathrm{NaOH} 0,5 \mathrm{~mol} \mathrm{I}^{-1}$ ). Du sulfite de sodium (solution à $0,2 \mathrm{~mol}^{-1}$ ) a été ajouté dans les échantillons de la deuxième série de façon à obtenir des concentrations initiales de 100 ou $1000 \mu \mathrm{mol} \mathrm{I}^{-1}$. Les échantillons de ces deux séries ont été stockés 2, 24 et 120 heures avant que leur concentration en TOX ne soit déterminée. Les échantillons de la troisième série ont été portés à $\mathrm{pH} 2,0 ; 8,5$ et 11,5 et ont reçu, immédiatement après 
l'ajustement du $\mathrm{pH}$, une quantité de sulfite (solution à $0,1 \mathrm{~mol} \mathrm{I}^{-1}$ ) correspondant à une concentration initiale de $100 \mathrm{mmol} \mathrm{l}^{-1}$. Leur concentration en TOX a été mesurée 2 heures après.

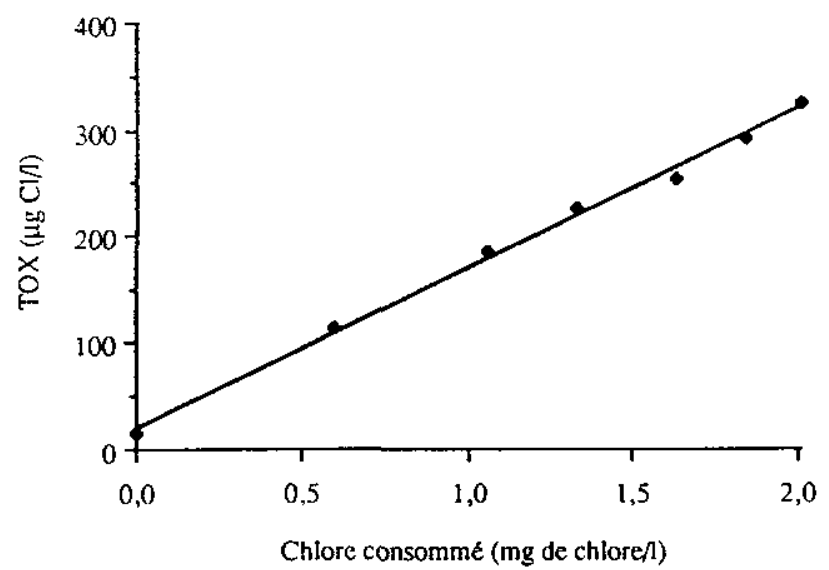

Figure 2 Evolution de la production de TOX en fonction du taux de chlore consommé $\left([\mathrm{SHA}]=5 \mathrm{mg} \mathrm{I}^{-1} ; \mathrm{pH}=6,1 ; 20 \pm 1^{\circ} \mathrm{C}\right.$; temps de réaction = 15 heures).

Evolution of TOX production versus chlorine consumption ([AHS] = $5 \mathrm{mg} r^{1} ; \mathrm{pH}=6.1 ; 20 \pm 1^{\circ} \mathrm{C} ; 15$ hours reaction time).

L'ensemble des résultats obtenus (tableau 1) montre que :

- un stockage dans un milieu de $\mathrm{pH}$ basique ou voisin de la neutralité de solutions chlorées de SHA dépourvues d'oxydant résiduel entraîne une diminution significative de la concentration en TOX (de l'ordre de $10 \%$ en 24 heures pour des $\mathrm{pH}$ de 6,1 à 8,5). Cette diminution est encore plus sensible lorsque le $\mathrm{pH}$ et/ou le temps de stockage augmentent ;

- l'addition de sulfite de sodium, aux concentrations initiales considérées, entraîne également une diminution significative de la concentration en TOX (de l'ordre de $10 \%$ en 2 heures pour des pH de 6,1 à 8,5). L'augmentation de la dose de sulfite ajoutée et/ou du temps de stockage contribue à accentuer ce phénomène ;

- l'addition de sulfite à une concentration initiale de $100 \mu \mathrm{mol} \mathrm{I}^{-1}$ dans des échantillons portés à différents pH entraîne, en 2 heures, une diminution de la concentration en TOX plus marquée que celle obtenue par la seule action du pH pendant le même laps de temps.

L'ensemble de ces résultats concorde avec ceux obtenus par SADIKI et al. (1990) à partir d'une eau de distribution chlorée et stockée à différents pH en l'absence d'oxydant résiduel, avec ou sans ajout de sulfite de sodium. D'autres auteurs ont également fait état d'une certaine labilité des composés organohalogénés en milieu neutre ou basique (CROUE et RECKHOW, 1989 ; KOPFLER et al., 1985 ; HOLMBOM et al., 1984 ; KRINGSTAD et al., 1983). En tant que réactif nucléophile (HENDRICKSON et al., 1970), l'ion hydroxyde peut 
conduire à des réactions de déshalogénation et comme le montrent les résultats obtenus, ces réactions sont favorisées en milieu basique.

Tableau 1 Influence du $\mathrm{pH}$, de l'addition de sulfite de sodium et du temps de stockage sur la concentration en TOX (Conditions de chloration : [SHA] $=5$ $\mathrm{mg} \mathrm{l}-1 ; \mathrm{pH}=6,1 ; 20 \pm 1^{\circ} \mathrm{C} ;\left[\mathrm{Cl}_{2}\right]_{0}=1,\left.13 \mathrm{mg} \mathrm{Cl}_{2}\right|^{-1}=0,23 \mathrm{mg} \mathrm{Cl}_{2} /$ mg SHA; $\left[\mathrm{Cl}_{2}\right] 15 \mathrm{~h}=0 \mathrm{mg} \mathrm{I} \mathrm{I}^{-}$; concentration moyenne en TOX après 15 heures de chloration $=204 \pm 4 \mu \mathrm{g} \mathrm{Cl} \mathrm{I-1}^{-1}$; stockage à $20 \pm$ $1{ }^{\circ} \mathrm{C}$ et à l'obscurité). La reproductibilité de la mesure est de $2 \%$.

Table 1 Effect of $\mathrm{pH}$, addition of sodium sulfite and storage time on the TOX concentration (Chlorination conditions: $[\mathrm{AHS}]=5 \mathrm{mg}^{-1} ; \mathrm{pH}=6.1$; $20 \pm 1^{\circ} \mathrm{C} ;\left[\mathrm{Cl}_{2}\right]_{0}=1.13 \mathrm{mg} \mathrm{Cl}{ }_{2} \digamma^{-1}=0.23 \mathrm{mg} \mathrm{Cl} / \mathrm{mg} \mathrm{AHS} ;[\mathrm{Cl} 2,15 \mathrm{~h}$ $=0 \mathrm{mg} \mathrm{t}^{-1}$; average TOX concentration after a 15 hours chlorination = $204 \pm 4 \mu \mathrm{g} \mathrm{Cl}{ }^{-1}$; storage at $20 \pm 1^{\circ} \mathrm{C}$ in the dark). The precision on the results is $2 \%$.

\begin{tabular}{|cccccc|}
\hline $\begin{array}{c}\text { Durée du stockage } \\
\text { (heures) }\end{array}$ & $\begin{array}{c}\text { [Sulfite] } \\
\left(\mu \mathrm{mol} \mathrm{1}^{-1}\right)\end{array}$ & $\mathrm{pH}=2, \mathbf{0}$ & $\mathrm{pH}=6,1$ & $\mathrm{pH}=8,5$ & $\mathrm{pH}=11,5$ \\
\hline \multirow{2}{*}{2} & 0 & 207 & 202 & 203 & $160(-22)^{\mathrm{a}}$ \\
& 100 & $201(-1)^{\mathrm{a}}$ & $182(-11)^{\mathrm{a}}$ & $186(-9)^{\mathrm{a}}$ & $146(-28)^{\mathrm{a}}$ \\
\hline \multirow{2}{*}{24} & 1000 & - & $181(-11)^{\mathrm{a}}$ & - & - \\
& 100 & $202(-1)^{\mathrm{a}}$ & $189(-7)^{\mathrm{a}}$ & $177(-13)^{\mathrm{a}}$ & $128(-37)^{\mathrm{a}}$ \\
& 1000 & - & $168(-18)^{\mathrm{a}}$ & - & - \\
\hline \multirow{2}{*}{120} & 0 & $187(-8)^{\mathrm{a}}$ & $170(-17)^{\mathrm{a}}$ & $131(-36)^{\mathrm{a}}$ & $98(-52)^{\mathrm{a}}$ \\
& 100 & - & $154(-25)^{\mathrm{a}}$ & - & - \\
& 1000 & - & $141(-31)^{\mathrm{a}}$ & - & - \\
\hline
\end{tabular}

a pourcentage de variation de la concentration en TOX (par a percentage of fluctuation of the TOX concentration from rapport à la référence de $204 \pm 4 \mu \mathrm{g} \mathrm{Cl}{ }^{-1}$. the reference value of $204 \pm 4 \mu \mathrm{g} \mathrm{CL}{ }^{-1}$.

La littérature mentionne par ailleurs l'existence d'un processus de déshalogénation induit par les dérivés du SIV et en particulier par le sulfite de sodium (BAUMAN et STENSTROM, 1989 ; CROUE et RECKHOW, 1989). Cette déshalogénation apparaît d'autant plus poussée que le $\mathrm{pH}$ est élevé. Ce phénomène peut être expliqué par le fait que les ions sulfite $\left(\mathrm{SO}_{3}{ }^{2-}\right.$; espèce prédominante à $\mathrm{pH}>7,2$; $\mathrm{pKa}$ du couple $\mathrm{HSO}_{3}-/ \mathrm{SO}_{3}{ }^{2}-=7,2$ ) sont plus nucléophiles que les ions bisulfite ( $\left.\mathrm{HSO}_{3}-\right)$, ces deux espèces chimiques étant elles-mêmes plus nucléophiles que l'acide sulfureux $\left(\mathrm{H}_{2} \mathrm{SO}_{3}\right)$ ou les ions hydroxyde (BETTERTON et al., 1988 ; CROUE et RECKHOW, 1989). Cependant, en milieu très basique $(\mathrm{pH}=11,5)$, la chute de la concentration en TOX observée peut être attribuée aux effets combinés des ions sulfite $\left(10^{-4} \mathrm{~mol} \mathrm{l}^{-1}\right)$ et hydroxyde (environ $3.10^{-3} \mathrm{~mol}^{-1}$ ). II faut enfin noter que pour les temps de stockage importants (120 heures) mis en cuvre au cours de cette étude, une fraction non négligeable du SIV peut être oxydée en SVI par l'oxygène dissous $\left(5.10^{-4}\right.$ mol $1^{-1}$ de SIV pour une concentration en oxygène dissous de $8 \mathrm{mg} \mathrm{|-1}^{-1}$ ) (WATTS et ADAMS, 1983 ; FULLER et CRIST, 1941). 


\subsubsection{Déchloration par le sulfite et concentration en TOX}

Pour étudier l'effet d'une déchloration (diminution du chlore libre résiduel) sur la concentration en TOX, les échantillons ont été chlorés à pH 6,1 à un taux de $4,14 \mathrm{mg} \mathrm{Cl}_{2} \mathrm{I}^{-1}$. Après 10 heures, la concentration en chlore résiduel était de $2,51 \mathrm{mg} \mathrm{Cl}_{2} 1^{-1}$. Le chlore résiduel a été neutralisé par ajout de thiosulfate de sodium sur deux échantillons qui ont alors été immédiatement acidifiés. Leur concentration en TOX a été prise comme référence (264 $\pm 5 \mu \mathrm{g}$ $\mathrm{Cl} \mathrm{I}^{-1}$; tableau 2). Les autres échantillons ont reçu des doses de sulfite de sodium correspondant à une déchloration partielle, stcechiométrique ou à l'ajout d'un excès de réducteur. Après 2 heures, le chlore libre résiduel a été mesuré puis neutralisé par du thiosulfate de sodium et tous les échantillons ont été acidifiés.

Tableau 2 Influence d'une déchloration par le sulfite de sodium sur la concentration en $\operatorname{TOX}\left([\mathrm{SHA}]=5 \mathrm{mg} \mathrm{I}-1 ; \mathrm{pH}=6,1 ; 20 \pm 1^{\circ} \mathrm{C} ;\left[\mathrm{Cl}_{2}\right]_{0}=4,14 \mathrm{mg}\right.$ $\mathrm{Cl}_{2} \mathrm{I}^{-1}=0,83 \mathrm{mg} \mathrm{Cl} / \mathrm{mg}$ SHA; $\left[\mathrm{Cl}_{2}\right]_{10 \mathrm{~h}}=2,51 \mathrm{mg} \mathrm{Cl}_{2} \mathrm{I}^{-1}$; concentration moyenne en TOX après 10 heures de chloration $=264 \pm$ $5 \mu \mathrm{g} \mathrm{Cl} \mathrm{I}^{-1}$; temps de contact en déchloration $=2$ heures). La reproductibilité des mesures est de $2 \%$.

Table 2 Effect of a dechlorination treatment with sodium sulfite on the TOX concen-tration ([AHS] $=5 \mathrm{mg}{ }^{-1} ; \mathrm{pH}=6.1 ; 20 \pm 1^{\circ} \mathrm{C} ;\left[\mathrm{Cl}_{2}\right]_{0}=$ $4.14 \mathrm{mg} \mathrm{Cl}_{2} \mathrm{r}^{-1}=0.83 \mathrm{mg} \mathrm{Cl} / \mathrm{mg} \mathrm{AHS} ;\left[\mathrm{Cl}_{2}\right]_{10 \mathrm{~h}}=2.51 \mathrm{mg} \mathrm{Cl}_{2} \mathrm{~F}^{-1}$; average TOX concentration after a 10 hours chlorination $=264 \pm 5 \mu \mathrm{gl}$ $r^{1}$; dechlorination time $=2$ hours). The precision on the results is $2 \%$.

\begin{tabular}{|c|c|c|c|c|c|}
\hline Echantillon & $\begin{array}{l}\text { Chlore résiduel } \\
\text { après } 10 \mathrm{~h} \\
\text { de chloration } \\
\text { (mg } \mathrm{CL}_{2} \mathrm{I}^{-1} \text { ) }\end{array}$ & $\begin{array}{c}{\left[\mathrm{Na}_{2} \mathrm{SO}_{3}\right]_{0}} \\
\left.\left(\mathrm{mg}^{-1}\right)^{-1}\right)\end{array}$ & $\begin{array}{c}\text { Taux de } \\
\text { déchloration } \\
{\left[\mathrm{SO}_{3}^{2-} \mathrm{l}_{0} / \mathrm{Cl}_{2}\right]_{0}} \\
\left(\mathrm{~mol} \mathrm{~mol}^{-1}\right)\end{array}$ & $\begin{array}{l}\text { Chlore résiduel } \\
\text { après } 2 \mathrm{~h} \text { de } \\
\text { déchloration } \\
\left(\left.\mathrm{mg} \mathrm{Cl}_{2}\right|^{-1}\right)\end{array}$ & $\begin{array}{c}\text { ToX après } 2 \mathrm{~h} \text { de } \\
\text { déchloration }(\mu \mathrm{g} \\
\mathrm{CI}^{-1} \mathrm{~F}^{-1}\end{array}$ \\
\hline 1 & 2,62 & - & 1 (thiosulfate) & 0 & 263 \\
\hline 2 & 2,57 & - & 1 (thiosulfate) & 0 & 264 \\
\hline 3 & 2.51 & 0,89 & 0,2 & 1,78 & $280(+6)^{a}$ \\
\hline 4 & 2,53 & 1,78 & 0,4 & 1,44 & $268(+2)^{a}$ \\
\hline 5 & 2.57 & 267 & 0,6 & 0,95 & $273(+3)^{a}$ \\
\hline 6 & 2,51 & 3,56 & 0,8 & 0,34 & $273(+3)^{a}$ \\
\hline 7 & 2,24 & 4,45 & 1,0 & 0 & $289(+9)^{a}$ \\
\hline 8 & 2,53 & 6,24 & 1,4 & 0 & $254(-4)^{\mathrm{a}}$ \\
\hline 9 & 2,51 & 12,5 & 2,8 & 0 & $240(-9)^{a}$ \\
\hline 10 & 2,51 & 62.4 & 14,0 & 0 & $234(-11)^{a}$ \\
\hline 11 & 2,53 & 124,7 & 28,0 & 0 & $229(-13)^{a}$ \\
\hline
\end{tabular}

a pourcentage de variation de la concentration en TOX ( $\mathrm{par}$ rapport a ta reférence de $264 \pm 5 \mu \mathrm{g} \mathrm{CI}{ }^{-1}$. a percentage of fluctuation of the TOX concentration from the reference value of $264 \pm 5 \mu \mathrm{g} \mathrm{CL} r^{-1}$.

Les résultats des mesures de concentrations en TOX ont été reportés dans le tableau 2 et sur la figure 3 . Sur cette dernière, le taux de déchloration a été représenté en échelle logarithmique. L'examen de ces résultats montre que :

- lors d'une déchloration partielle, les concentrations en TOX mesurées 2 heures après l'ajout du sulfite sont supérieures à celles des échantillons 
pris comme référence (jusqu'à $6 \%$; figure 3). Ce phénomène peut être expliqué par le fait que la demande en chlore des solutions de SHA n'est pas satisfaite après 10 heures. Le chlore libre encore présent après addition du sulfite continue donc à réagir sur la matière organique pour conduire à la

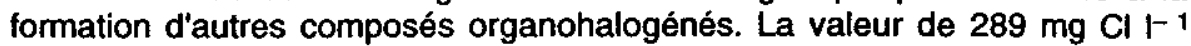
obtenue pour une déchloration stœchiométrique semble anormalement élevée ; ceci peut provenir d'une consommation en chlore en 10 heures supérieure à celle observée pour les autres échantillons $\left(2,24 \mathrm{mg} \mathrm{Cl}_{2}{ }^{-1}\right.$ de chlore résiduel après 10 heures de chloration contre $2,54 \mathrm{mg} \mathrm{Cl} \mathrm{F}^{-1}$ pour l'ensemble des autres échantillons). Les teneurs en chlore résiduel relevées après 2 heures de déchloration partielle permettent de confirmer la valeur de 1 mole/mole pour la stœchiométrie de la réaction d'oxydo-réduction entre le sulfite de sodium (formes $\mathrm{SO}_{3}{ }^{2}$ - et $\mathrm{HSO}_{3}{ }^{-}$en solution à $\mathrm{pH}$ neutre) et le chlore libre (formes $\mathrm{ClO}^{-}$et $\mathrm{HClO}$ en solution à pH neutre) (SADIKI et al., 1990 ; WHITE, 1986). Cette réaction conduit à la formation d'une mole d'ions chlorure et d'une mole d'ions sulfate par mole de sulfite ou de chlore consommée ;

- l'ajout d'un excès de sulfite conduit à la réduction de la concentration en TOX. L'ampleur de ce phénomène est d'autant plus grande que l'excès initialement introduit est important (figure 3) sans cependant dépasser 10 à $15 \%$ de la référence pour les plus fortes doses utilisées et à pH 6,1.

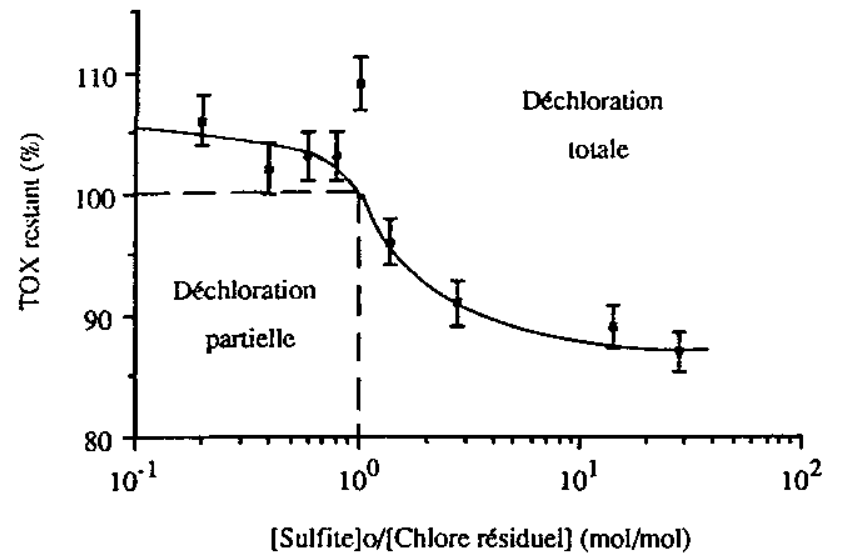

Figure 3 Influence d'une déchloration partielle ou totale sur la concentration en TOX. $100 \%$ de TOX restant correspond a une concentration de $264 \pm$ $5 \mu \mathrm{g} \mathrm{Cl} \mathrm{F}^{-1}\left([\mathrm{SHA}]=5 \mathrm{mg} \mathrm{I}-1 ; \mathrm{pH}=6,1 ; 20 \pm 1^{\circ} \mathrm{C} ;\left[\mathrm{Cl}_{2}\right]_{0}=4,14\right.$ $\mathrm{mg} \mathrm{Cl}{ }^{-1}=0,83 \mathrm{mg} \mathrm{Cl} / \mathrm{mg} S \mathrm{HA} ;\left[\mathrm{Cl}_{2}\right]_{10 \mathrm{~h}}=2,51 \mathrm{mg}^{2} \mathrm{Cl}_{2} \mathrm{~F}^{-1}$; temps de déchloration $=2$ heures).

Effect of a partial or complete dechlorination treatment on the TOX concentration. $100 \%$ of TOX corresponds to a concentration of $264 \pm$ $5 \mu \mathrm{gCl} \mathrm{F}^{-1}$ ([AHS] $=5 \mathrm{mgt} ; \mathrm{pH}=6.1 ; 20 \pm 1^{\circ} \mathrm{C}$; $\left[\mathrm{Cl}_{2}\right]_{0}=4.14 \mathrm{mg} \mathrm{Cl}_{2} \mathrm{r}^{-1}=0.83 \mathrm{mg} \mathrm{Cl} / \mathrm{mg} \mathrm{AHS} ;\left[\mathrm{Cl}_{2}\right]_{10 \mathrm{~h}}=2.51 \mathrm{mg}$ $\mathrm{Cl}_{2}{ }^{2}{ }_{1} ; 2$ hours dechlorination time). 
Ces résultats concordent avec ceux obtenus par SADIKI et al. (1990) à partir d'une eau potable, à savoir : pas de réduction de la concentration en TOX par déchloration partielle et diminution significative mais toutefois limitée de cette concentration pour des taux de traitement en sulfite de sodium importants.

\subsection{Déchloration par le sulfite, concentration en TOX et activité mutagène}

Six échantillons de 44 litres d'une solution de SHA à $15 \mathrm{mg} \mathrm{t}^{-1}$ (COD $=7,5$ $\mathrm{mg} \mathrm{Cl} \mathrm{I}^{-1} ; \mathrm{pH}=6,9 ; \mu=0,01 \mathrm{~mol}^{-1}$ ) ont été chlorés à un taux de $0,67 \mathrm{mg}$ $\mathrm{Cl}_{2} / \mathrm{mg}$ SHA (soit $10,0 \mathrm{mg} \mathrm{Cl} \mathrm{I}^{-1}$ ). Après 18 heures de réaction, la concentration moyenne en chlore libre était de $4,0 \pm 0,\left.1 \mathrm{mg} \mathrm{Cl}_{2}\right|^{-1}$. Le chlore résiduel a été neutralisé par du thiosulfate de sodium sur un échantillon qui a été ensuite acidifié. Sa concentration en TOX et son activité mutagène ont été prises comme références. Les autres échantillons ont reçu du sulfite de sodium (solutions à 0,05 ou $0,1 \mathrm{~mol}^{-1}$ ) à des doses correspondant à une déchloration partielle, stœchiométrique ou à l'ajout d'un excès de réducteur. Des prélèvements ont été effectués avant et après l'addition du sulfite pour déterminer les concentrations en TOX. Après 3,5 heures, le chlore résiduel a été neutralisé par du thiosulfate de sodium. Tous les échantillons ont été immédiatement acidifiés à pH 2,0. Après extraction sur résines XAD et reconcentration, l'activité mutagène a été évaluée sur les extraits obtenus.

En raison du nombre d'étapes nécessaires à la préparation d'un extrait, seulement 3 échantillons ont pu être menés de front. C'est pourquoi le tableau 3 fait apparaître deux séries distinctes de résultats qui seront par conséquent interprétés séparément.

Les concentrations en TOX mesurées (tableau 3) confirment les résultats précédents (tableau 2):

- une déchloration partielle entraîne une augmentation de la concentration en TOX ;

- l'ajout d'un excès de sulfite conduit à une diminution de la concentration en TOX, diminution toutefois limitée (moins de $7 \%$ pour le plus fort excès de réducteur).

Les réponses au test de mutagénèse obtenues pour la souche TA100 sans activation métabolique (tableau 3 ; l'ensemble des réponses des deux souches avec ou sans $S_{9}$ mix a été détaillé par ailleurs : MORLAY et al., 1991) montrent que :

- une déchloration partielle ne diminue pas de façon significative l'activité mutagène des échantillons (moins de $10 \%$ pour les échantillons $n^{\circ} 2$ et 3 comparés à l'échantillon $n^{\circ} 1$ );

- l'ajout d'un excès de sulfite permet de réduire notablement l'activité mutagène (échantillons $n^{\circ} 5$ et 6 comparés à l'échantillon $n^{\circ} 4$ ). Cette diminution est d'autant plus prononcée que l'excès de sulfite est important; elle atteint $50 \%$ pour les conditions opératoires choisies.

La comparaison des résultats fournis par détermination de l'activité mutagène et de la concentration en TOX (figure 4) indique que la diminution de la génotoxicité observée après ajout d'un excès de sulfite de sodium serait 
due à l'action de celui-ci sur des composés mutagènes non halogénés ou sur des molécules halogénées hautement mutagènes présentes à l'état de traces et qui ne représenteraient par conséquent qu'une très faible part de la concentration en TOX. L'absence de corrélation entre activité mutagène et concentration en composés organohalogénés totaux avait déjà été rapportée par FAWELL et FIELDING (1985) et FAWELL et al. (1986), ces auteurs ayant toutefois étudié l'étape de chloration et non pas celle de déchloration.

Tableau 3 influence d'une déchloration partielle (échantillons $n^{\circ} 1,2$ et 3 ) ou totale (échantillons $n^{\circ} 4,5$ et 6) sur la concentration en rOX et l'activité mutagène (TA 100 sans $S_{9}$ mix) ([SHA] $=15 \mathrm{mg} \mathrm{l}^{-1} ; \mathrm{pH}=6,9 ; 20 \pm$ $1^{\circ} \mathrm{C} ;\left[\mathrm{Cl}_{2}\right]_{0}=10,0 \mathrm{mg} \mathrm{Cl} \mathrm{I}^{-1}=0,67 \mathrm{mg} \mathrm{Cl} 2 / \mathrm{mg} \mathrm{SHA} ;\left[\mathrm{Cl}_{2}\right]_{18 \mathrm{~h}}=$ $4,0 \mathrm{mg} \mathrm{Cl}_{2}{ }^{-1}$; ternps de contact en déchloration $=3,5$ heures).

Table 3 Effect of a partial (samples $n^{\circ} 1,2$ and 3) or complete (samples $n^{\circ} 4,5$ and 6) dechlorination on the TOX concentration and on the mutagenic activity (TA 100 without $S_{g} \mathrm{mix}$ ) ([AHS] $=15 \mathrm{mg}^{-1} ; \mathrm{pH}=6.9 ; 20 \pm$ $1^{\circ} \mathrm{C} ;\left[\mathrm{Cl}_{2}\right]_{0}=10.0 \mathrm{mg} \mathrm{Cl}{ }_{2} \mathrm{I}^{1}=0.67 \mathrm{mg} \mathrm{Cl} / \mathrm{mg} \mathrm{AHS} ;\left[\mathrm{Cl}_{2}\right] 1_{8 \mathrm{~h}}=$ $4.0 \mathrm{mg} \mathrm{Cl}_{2} \vdash^{-1}$; dechlorination time $=3.5$ hours).

\begin{tabular}{|c|c|c|c|c|c|c|}
\hline Echantillon & 1 & 2 & 3 & 4 & 5 & 6 \\
\hline $\begin{array}{l}\text { Taux de déchloration } \\
{\left[\mathrm{S}_{\mathrm{rvl}} /\left[\mathrm{Cl}_{2}\right]_{0} \text { (mol mol-1) }\right.}\end{array}$ & $\begin{array}{c}1 \\
\text { (thiosulfate) }\end{array}$ & 0,91 & 0,98 & 1 & 20 & 10 \\
\hline $\begin{array}{l}\text { Chlore résiduel }\left(\mathrm{mg} \mathrm{CL}_{2} \mathrm{r}^{-1}\right) \\
\text { et TOX }\left(\mu \mathrm{g} \mathrm{Cl}{ }^{-1}\right) \text { après } 18 \mathrm{~h} \text { de } \\
\text { chloration }\end{array}$ & $\begin{array}{l}4,22 \\
1205\end{array}$ & $\begin{array}{l}3,99 \\
1280\end{array}$ & $\begin{array}{l}4,12 \\
1218\end{array}$ & $\begin{array}{r}4,08 \\
949\end{array}$ & $\begin{array}{r}4,07 \\
949\end{array}$ & $\begin{array}{l}3,98 \\
937\end{array}$ \\
\hline $\begin{array}{l}\text { Chlore résiduel }\left(\left.\mathrm{mg} \mathrm{CL}_{2}\right|^{-1}\right) \\
\text { et TOX }\left(\left.\mu \mathrm{g} \mathrm{Cl}\right|^{-1}\right) \text { après } 3,5 \mathrm{~h} \text { de } \\
\text { déchloration }\end{array}$ & $\underline{0}$ & $\begin{array}{l}0,35 \\
1567\end{array}$ & $\begin{array}{l}0,10 \\
1468\end{array}$ & $\begin{array}{c}0 \\
928\end{array}$ & $\begin{array}{c}0 \\
879\end{array}$ & $\begin{array}{c}0 \\
885\end{array}$ \\
\hline $\begin{array}{l}\text { Pentes calculées : TA100 } \\
\left.\text { sans S9mix (révertants } \Gamma^{\prime}\right)\end{array}$ & 3691 & $\begin{array}{l}3350 \\
(-9)^{a}\end{array}$ & $\begin{array}{l}3612 \\
(-2)^{a}\end{array}$ & 3052 & $\begin{array}{l}2269 \\
(-26)^{a}\end{array}$ & $\begin{array}{l}1629 \\
(-47)^{3}\end{array}$ \\
\hline
\end{tabular}

a pourcentage de réduction de f'activité mutagène par rapport a la référence (échantilions $n^{\circ} 1$ et 4 pour ta première et la deuxième séries respectivement). a percentage of fluctuation of the mutagenic activity comapred to the reference value (samples $n^{\circ} 1$ and 4 for the first and the second series respectively).

Les résultats du test d'AMES obtenus ici à partir de solutions de SHA chlorées sont en désaccord avec ceux obtenus par WILCOX et DENNY (1985) ou FAWELL et al. (1986) sur des eaux de consommation. Ces auteurs ont observé une diminution de l'activité mutagène d'une eau de distribution chlorée par suite d'une déchloration partielle avec du sulfite de sodium, à condition toutefois que celle-ci conduise à une concentration en chlore résiduel inférieure à $0,5 \mathrm{mg} \mathrm{t}^{-1}$. Toutefois, FAWELL ot al. (1986) ont observé qu'une déchloration partielle avec une concentration en chlore résiduel inférieure à $0,5 \mathrm{mg} \mathrm{I}^{-1}$ n'affectait pas le taux d'activité mutagène de solutions d'acide humique chlorées alors qu'elle semblait détruire une partie de l'activité d'eaux de distribution chlorées. Une explication possible de ce phénomène consisterait, selon ces auteurs, en l'existence de précurseurs différents selon l'origine 

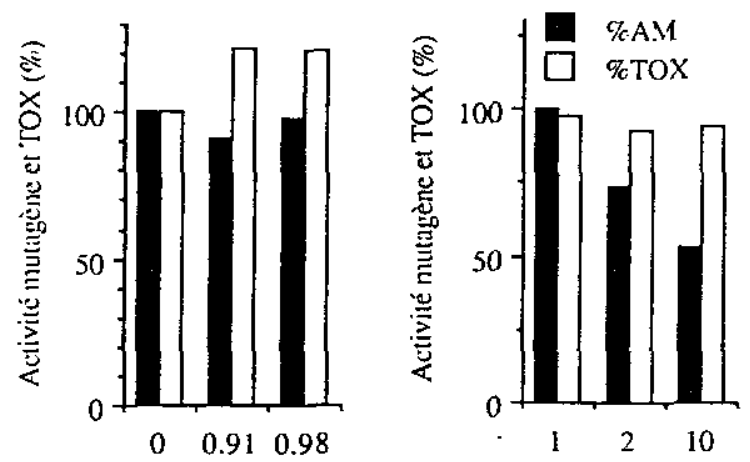

Taux de déchloration ([sulfite]//chlore résiduel] en mol mol-1)

Figure 4 Influence d'une déchloration partielle ou totale sur le taux d'activité mutagène et la concentration en TOX. Déchloration partielle : $100 \%$ correspond à l'activité mutagène de l'échantillon non déchloré (échantit Ion $n^{\circ} 1$; tableau 3 ). Déchloration totale : $100 \%$ correspond a l'activité mutagène de l'échantillon déchloré stcechiométriquement par le sulfite (échantillon $n^{\circ} 4$; tableau 3). Concentration en TOX : $100 \%$ correspond, pour chaque échantillon, à la valeur avant déchloration ([SHA] = $15 \mathrm{mg} \mathrm{l}^{-1} ; \mathrm{pH}=6,9 ; 20 \pm 1^{\circ} \mathrm{C} ;\left[\mathrm{Cl}_{2}\right]_{0}=10,0 \mathrm{mg} \mathrm{Cl}^{2} \mathrm{I}^{-1}=0,67$ $\left.\mathrm{mg} \mathrm{Cl} / \mathrm{mg} \mathrm{SHA} ;\left[\mathrm{Cl}_{2}\right]_{18 \mathrm{~h}}=4,0 \mathrm{mg} \mathrm{Cl} 2\right\}^{-1} ;$ temps de déchloration = 3,5 heures).

Effect of a partial or complete dechlorination treatment on the mutagenic activity level and on the TOX concentration. Partial dechlorination: $100 \%$ value corres-ponds to the mutagenic activity of the non dechlorinated sample (sample $n^{\circ} 1$; table 3 ). Complete dechlorination : $100 \%$ value corresponds to the mutagenic activity of the sample dechlorinated with a stoichiometric dose of sulfite. TOX concentration: $100 \%$ value corresponds, for each sample, at the TOX concentration before the dechlorination treatment (sample $n^{\circ} 4$; table 3) ([AHS] $=15$ $\mathrm{mg}{ }^{1} ; \mathrm{pH}=6.9 ; 20 \pm 1^{\circ} \mathrm{C} ;\left[\mathrm{Cl}_{2}\right]_{0}=10.0 \mathrm{mg} \mathrm{Cl} \mathrm{I}^{-1}=0.67 \mathrm{mg}$ $\mathrm{Cl}_{2} / \mathrm{mg}$ AHS ; $\left.\left[\mathrm{Cl}_{2}\right]_{18 \mathrm{~h}}=4.0 \mathrm{mg} \mathrm{Cl}{ }_{2}\right\}^{2} ; 3.5$ hours dechlorination time).

de l'eau étudiée, précurseurs qui, par chloration, donneraient naissance à des composés mutagènes susceptibles ou non d'être détruits lors d'une déchloration. Cependant, une réduction de la génotoxicité d'échantillons aqueux chlorés (eaux de surface ou de distribution, solutions de SHA...) après un traitement de déchloration par les dérivés du soufre tétravalent ne peut être obtenue que si les vitesses de réaction entre le SIV et les composés mutagènes permettent une dégradation effective de ces derniers dans les conditions du traitement ( $\mathrm{pH}$, temps de contact, dose de sulfite). Dans le cas particulier d'une déchloration partielle, en raison d'une part de la très grande vitesse de réaction entre les dérivés du SIV et le chlore libre résiduel (réaction quasiinstantanée ; WHITE, 1986 ; FOGELMAN et al., 1989) et d'autre part du rapport de concentrations existant entre le chlore libre résiduel $\left(0,2\right.$ à $\left.1 \mathrm{mg} \mathrm{l}^{-1}\right)$ et les composés mutagènes présents à l'état de traces (de l'ordre du ng $\mathbf{~ I ~}^{-1}$ voire de quelques dizaines de $\mathrm{ng}(-1)$, une diminution de l'activité mutagène semble extrêmement peu probable. Compte tenu des lois de cinétique compétitive et des valeurs des constantes de vitesse pour la réaction des ions 
sulfite sur des composés organiques (SADIKI et al., 1990 ; CROUE et RECKHOW, 1989), les traitements de déchloration partielle dans les conditions mises en œuvre dans le cadre du traitement des eaux à potabiliser conduisent à une consommation très rapide de l'agent réducteur par le chlore libre résiduel et ne permettent pas, comme le confirme cette étude, d'obtenir une diminution de la concentration en composés organohalogénés ou une réduction de la génotoxicité. Sur le plan pratique, il convient cependant de noter que l'évaluation de l'activité mutagène d'échantillons chlorés de SHA en solution aqueuse très diluée ou d'eaux de distribution est particulièrement délicate. En effet, les étapes d'extraction des composés mutagènes sur résines XAD (adsorption, élution), de reconcentration des extraits ainsi que les risques de modification de la structure chimique des composés mutagènes au cours de ces différentes phases (hydrolyse possible lors du stockage de l'eau, chauffage lors de la reconcentration) influent sur la reproductibilité des résultats. Ces réserves doivent être prises en compte au niveau de l'interprétation des résultats expérimentaux ainsi qu'à l'égard des données bibliographiques pour lesquelles les conditions opératoires retenues ne sont pas toujours suffisamment détaillées.

\section{4 - CONClUSION}

Les résultats obtenus au cours de cette étude à partir de solutions de SHA a $5 \mathrm{mg} \mathrm{I}^{-1}\left(C O D=2,5 \mathrm{mg} \mathrm{Ct} \mathrm{t}^{1}\right)$ ont permis de montrer que les composés organohalogénés formés lors de la chloration de substances humiques d'origine aquatique (taux d'incorporation du chlore dans les molécules organiques de l'ordre de $0,15 \mathrm{mg} / \mathrm{mg}$ de chlore consommé à $\mathrm{pH} 6,1)$ peuvent être dégradés, soit par hydrolyse lors du stockage des échantillons en milieu neutre (10\% d'abattement du paramètre TOX en 24 heures à pH 6,1-8,5) ou basique (20\% de diminution après 2 heures à pH 11,5), soit par action des dérivés oxygénés du soufre tétravalent (ions sulfite et bisulfite). Dans ce demier cas, la réduction de la concentration en composés organohalogénés ne peut cependant être obtenue que pour des doses de sulfite mises en œuvre correspondant au moins à une élimination totale du chlore libre résiduel. D'autre part, elle dépend du temps de réaction, du pH et de l'excès initial de sulfite de sodium. Par ailleurs, la réduction de la concentration en composés organohalogénés observée à un pH donné est plus importante en présence qu'en l'absence de sulfite.

L'étude réalisée sur des solutions de SHA à $15 \mathrm{mg} \mathrm{I}^{-1}$ (COD = 7,5 mg C $\vdash^{1}$ ) a permis de suivre l'évolution de l'activité mutagène parallèlement à celle de la concentration en TOX Jors d'une déchloration à pH neutre. Il ressort des résultats obtenus qu'une déchloration partielle ne diminue pas de manière significative lactivité mutagène des échantillons (moins de $10 \%$ ) alors que l'ajout d'une dose initiale de sulfite équivalente à deux fois la stcechiométrie de neutralisation du chlore résiduel entraîne une réduction de $30 \%$ environ de la génotoxicité. Cette réduction peut atteindre $50 \%$ pour une dose initiale de sulfite introduit de 10 fois la stcechiométrie. La comparaison de l'évolution 
de lactivité mutagène et de celle de la concentration en TOX semble indiquer que la disparition d'une partie de la génotoxicité des échantillons par déchloration totale serait due à l'action du sulfite sur des composés mutagènes non halogénés ou sur des composés halogénés hautement mutagènes mais présents à l'état de traces. Ceci expliquerait l'abattement de $50 \%$ de la génotoxicité pour une réduction de moins de $7 \%$ de la concentration en TOX.

Sur le plan pratique, ces résultats obtenus au laboratoire à partir de solutions aqueuses de substances humiques d'origine aquatique indiquent que les traitements de déchloration partielle par les dérivés du soufre tétravalent mis en ceuvre au niveau de certaines unités de production d'eau potable ne peuvent diminuer de manière significative, ni la teneur globale en composés organohalogénés (paramètre TOX), ni la génotoxicité de l'eau. Les expériences actuellement réalisées à partir d'échantillons d'eau en cours de potabilisation ont pour but de confirmer les résultats obtenus lors de cette étude.

\section{RÉFÉRENCES BIBLIOGRAPHIQUES}

ANSELME C., MALLEVIALLE J., BORDET J.P., 1987. Les problèmes de mauvais goûts de l'eau potable. Construction d'un modèle prédictif. T.S.M., 6, 261-272.

BAUMAN L., STENSTROM M.K., 1989. Observations of the reaction between organohalides and sulfite. Environ. Sci. Technol., 23, 232-236.

BELLAR T.A., LICHTENBERG J.J., 1974. Determining volatile organics at microgramper-liter level by gas chromatography. $J$. Am. Water Works Assn., 66, 739-744.

BETTERTON E.A., EREL Y., HOFFMAN M.R., 1988. Aldehyde-bisulfite adducts : prediction of some of their thermodynamic and kinetic properties. Environ. Sci. Technol., 22, 92-99.

CHEH A.M., SKOCHDOPOLE J., HEILIG C., KOSKI P.M., COLE L.,1980. Destruction of direct-acting mutagens in drinking water by nucleophiles : implications for mutagen identification and mutagen elimination from drinking water. In Water Chlorination; Environmental impact and Health Effects, Jolley ot al. (Eds.), Ann Arbor Science Publishers, Michigan, 3, 803-815.

CROUE J.P.,1987. Contribution à l'étude de roxydation par le chlore et l'ozone dracides fulviques naturels extraits d'eaux de surface. Thèse de doctorat, Université de Poitiers, 9 juillet 1987, $\mathrm{n}^{\circ}$ d'ordre 89.
CROUE J.P., RECKHOW D.A., 1989. Destruction of chlorination byproducts with sulfite. Environ. Sci. Technol., 23, 1412-1419.

FAM S.A., 1986. Non-volatile chlorinated organics produced during disinfection of water : analysis, formation and minimization of their production. Chap. 5: Effect of dechlorinating agents. Thèse de doctorat, Université de Californie à Los Angeles, CA.

FAWELL J.K., FIELDING M., 1985. Identification and assessment of hazardous compounds in drinking water. The Science of the Total Environment, Elsevier Science Publishers B.V., Amsterdam, 47, 317-341.

FAWELL J.K., FIELDING M., HORTH H., JAMES H.A., LACEY R.F., RIDGWAY J.W., WILCOX P., WILSON I., 1986. Health aspects of organics in drinking water. Technical Report TR 231, Water Research Centre, Medmenham, Marlow, Bucks., England.

FIELDING M., HORTH H., 1986. Formation of mutagens and chemicals during water treatment chlorination. Wat. Supply, 4, 103126.

FOGELMAN K.D., WALKER D.M., MARGERUN D.W., 1989. Non-metal redox kinetics: hypochlorite and hypochlorous acid reactions with sulfite. Inorg. Chem., 28 , 986-993. 
FULLER E.C., CRIST R.H., 1941. The rate of oxidation of sulfite ions by oxygen. J. Am. Chem. Soc., 63,1644-1650.

GLAZE W.H., PEYTON G.R., SELEH F.Y.,1979. Analysis of disinfection byproducts in water and waste-water. Int. $J$. Environ. Anal. Chem., 7, 143-160.

GLAZE W.H., SELEH F.Y., KINSTLEY W., 1980. Characterization of non-volatile halogenated compounds formed during water chlorination. In: Water Chlorination; Environmental Impact and Health Effects, Jolley et al. (Eds.), Ann Arbor Science Publishers, Michigan, 3, 99-108.

HENDRICKSON J.B., CRAM D.J., HAMMOND G.S., 1970. Organic Chemistry. Third edition, McGraw-Hill Series in Undergraduate Chemistry.

HOLMBOM B., VOSS R.H., MORTIMER R.D., WONG A., 1984. Fractionation, isolation and characterization of Ames mutagenic compounds in kraft chlorination effluents. Environ. Sci. Technol., 18, 333-337.

HORTH H., 1989. Identification of mutagens in drinking water. Aqua, $38: 80-100$.

JESTIN J.M., LEVY Y., HOTELIER J., 1986. Les odeurs générées par la chloration. Identification du problème, conséquences pour le traitement. Journées Information Eaux, Tome 1, conférence 3, Poitiers, septembre 1986.

KOPFLER F.C., RINGHAND H.P., COLEMAN W.E., MEIER J.R., 1985. Reactions of chlorine in drinking water, with humic acid and in vivo. In : Water Chlorination: Chemistry, Environmental Impact and Health Effects, Jolley et al. (Eds.), Lewis Publishers, Michigan, 5, 161-173.

KRINGSTAD K.P., LJUNGQUIST P.O., DE SOUSA F., STROMBERG L.M., 1983. On the formation of mutagens in the chlorination of humic acid. Environ. Sci. Technol., 17, 553-555.

MAQUENNEHAN F., CLAUSE J., 1987. Chloration et déchloration des eaux de Paris. L'Eau, I'Industrie, les Nuisances, $113,63-66$.

MARON D.M., AMES B.N., 1983. Revised methods for the Saimonella mutagenicity test. Mutat. Res., 113, 173-215.
MEIER J.R , LINGG R.D., BULL R.J., 1983. Formation of mutagens following chlorination of humic acid; a model for mutagen formation during drinking water treatment. Mutat. Res., 118, 25-41.

MORLAY C., DE LAAT J., DORE M., COURTOIS Y., FESTY B., HOUEL N., MONTIEL A. (1991) Effect of an addition of sodium sulfite on the mutagenicity of chlorinated solutions of aquatic humic substances. Bull. Environm. Contam. Toxicol., 47, 15-22.

OLIVER B.G., 1978. Chlorinated non-volatile organics produced by the reaction of chlorine with humic materials. Cancer Res., 11, 21-22.

ROOK J.J.,1974. Formation of haloforms during chlorination of natural water. Water Treatment and Exam., 23, 234-243.

SADIKI A., DE LAAT J., DORE M., MONTIEL A., HOUEL N., 1990. La déchloration des eaux potables : étude de la réactivité des dérivés du soufre IV sur les composés organiques en milieu aqueux dilué. J.F. Hydrol., 21, 77-92.

TREHY M.L., BIEBER T.I., 1981. Detection, identification and quantitative analysis of dihaloacetonitriles in chlorinated natural waters. In : Advances in the Identification and Analysis of Organic Pollutants in Water, Keith L.H. Ed., Ann Arbor Science, Ann Arbor, MI, 2, 941-975.

THURMAN E.M., MALCOLM R.L., 1981. Preparative isolation of aquatic humic substances. Environ. Sci. Technol., 15, 463-466.

WATTS R.J., ADAMS V.D., 1983. The elimination of sulfur dioxide interference in the low level chemical oxygen demand analysis. Water Res., 17, 715-718.

WHITE G.C., 1986. The handbook of chlorination, second edition. Van Nostrand Reinhold Company Publisher, New York, 755-788.

WILCOX P., DENNY S., 1985. Effect of dechiorinating agents on the mutagenic activity of chlorinated water samples. In : Water Chlorination: Chemistry, Environmental Impact and Health Effects, Jolley et al. (Eds.), Lewis Publishers, Michigan, 5, 1341-1353. 